\title{
Hallazgos en la angiografía por tomografía de coherencia óptica en pacientes mexicanos con telangiectasias maculares tipo 2
}

\section{Optical coherence tomography angiography findings in macular telangiectasia type 2 in Mexican patients}

\author{
Paola Brito-Sandoval* y Sergio Rojas-Juárez \\ Departamento de Retina y Vitreo, Fundación Hospital Nuestra Señora de la Luz, Institución de Asistencia Privada, Ciudad de México, México
}

\begin{abstract}
Resumen
Objetivo: Describir por medio de la angiografía por tomografía de coherencia óptica (OCT-A) las características de los capilares perifoveales, plexos afectados y cambios de la retina neurosensorial en telangiectasias maculares idiopáticas y ver su relación con la agudeza visual. Método: Estudio descriptivo y analítico, donde se realizaron OCT-A Heidelberg en pacientes diagnosticados con telangiectasias maculares tipo 2. Resultados: Se estudiaron 43 ojos de 24 pacientes adultos. Edad promedio 60 años. El $80 \%$ bilateral. Pacientes diabéticos con agudeza visual significativamente peor $(p<0.05)$. Según los cambios vasculares, se encontró en estadio 1 el 21\%, en el 2 el 38\%, en 3 el $32 \%$ y en el 4 el 9\%. La localización más frecuente de los cambios vasculares en la OCT-A con respecto a la fóvea fueron temporales (63\%), seguidos por superiores $(19 \%)$. No hay relación entre cuadrante y agudeza visual $(p=0.25)$. La afección más frecuente fue el plexo profundo $(94 \%)$. La morfología de los vasos en el sitio de la lesión fue un engrosamiento discreto, dilataciones, y en el $33 \%$ de los casos se observaron lesiones aneurismáticas, neovascularizaciones, en el $21 \%$ (el $55 \%$ de ellas fueron subclínicas y únicamente detectadas con OCT-A). No se encontró relación entre el grosor macular y la agudeza visual ( $p=0.29)$. Conclusiones: Encontramos pacientes en los diferentes estadios de la enfermedad. El $21 \%$ presentó enfermedad avanzada con neovascularización y el 55\% presentó neovascularización subclínica, no detectable en fluorangiografía y OCT regular. El OCT-A es el estudio ideal para diagnosticar esta entidad sin necesidad de otros estudios.
\end{abstract}

Palabras clave: Idiopática. Mácula. Telangiectasias. Tomografía de coherencia óptica.

\section{Abstract}

Purpose: To describe the characteristics of perifoveal capillaries, the plexuses involved and changes of the neurosensory retina in idiopathic macular telangiectasia using optical coherence tomography angiography (OCT-A) and correlate these findings with visual acuity. Method: Descriptive and analytical study. Heidelberg OCT-A was performed in patients diagnosed with Type 2 macular telangiectasia. Results: A total of 43 eyes of 24 adult patients were studied. Average age was of 60 years, and $80 \%$ of the cases were bilateral. Diabetic patients had significantly worse visual acuity $(p<0.05)$. Regarding vascular changes, we found 21\% in Stage 1,38\% in Stage 2, 32\% in Stage 3 and 9\% in Stage 4. The most frequent location

\section{Correspondencia:}

*Paola Brito-Sandoval

Ezequiel Montes, 135

Col. Tabacalera, Del. Alcaldía Cuauhtémoc $\quad$ Fecha de recepción: 19-06-2019

C.P. 06030, Ciudad de México, México Fecha de aceptación: 25-11-2019

E-mail: polabs_1@ hotmail.com

DOI: 10.24875/RMO.M20000108

Disponible en internet: 01-03-2020

Rev Mex Oftalmol. 2020;94(2):77-82

www.rmo.com.mx

0187-4519/๑ 2019 Sociedad Mexicana de Oftalmología. Publicado por Permanyer. Este es un artículo open access bajo la licencia CC BY-NC-ND (http://creativecommons.org/licenses/by-nc-nd/4.0/). 
of vascular changes by OCT-A with respect to the fovea was temporal (63\%), followed by superior (19\%). There was no relationship between the affected quadrant and visual acuity $(p=0.25)$. The deep capillary plexus was affected in $94 \%$. We found discrete thickening, dilated vessels and aneurysmal lesions. Twenty-one percent had neovascularization, $55 \%$ of these were subclinical and only detected by OCT-A. No relationship was observed between macular thickness and visual acuity $(p=0.29)$. Conclusions: We found patients in different stages of the disease; $21 \%$ had advanced disease with neovascularization and $55 \%$ had subclinical neovascularization, not detectable by fluorescein angiography and regular OCT. OCT-A is the ideal study to diagnose this disorder without the need for further studies.

Key words: Idiopathic. Macular. Telangiectasia. Optical coherence tomography.

\section{Introducción}

Las telangiectasias maculares (MacTel) se caracterizan por anormalidades en los capilares del polo posterior. Hay dos formas bien definidas: las de tipo 1, congénitas o del desarrollo, usualmente unilaterales, son telangiectasias aneurismáticas y pueden ser parte de la enfermedad de Coats; y las de tipo 2, adquiridas del adulto, son usualmente bilaterales y también llamadas telangiectasias yuxtafoveolares ${ }^{1}$.

Las MacTel tipo 2 son una condición degenerativa con alteración de los capilares maculares y atrofia de la retina neurosensorial de etiología idiopática. Su prevalencia se ha reportado entre el 0.06 y el $0.1 \%$, y la edad promedio de presentación es de 63 años $^{1,2}$.

Las lesiones vasculares comienzan temporal a la mácula y subsecuentemente afectan la fóvea. Se afecta el plexo superficial y profundo.

Histopatológicamente, se ha encontrado pérdida de las células de Müller perifoveales y en los capilares retinianos hay proliferación de la membrana basal hacia el lumen, reducción del número de pericitos y áreas focales de disrupción de células endoteliales ${ }^{3}$.

Evidencias recientes apuntan a un rol central de las células de Müller. Los cambios encontrados en la autofluorescencia y la tomografía de coherencia óptica (OCT) preceden a los cambios vasculares en la fluorangiografía retiniana $(F A R)^{4}$.

En la FAR se observan capilares dilatados y ectásicos. Las zonas de hiperfluorescencia por fuga de contraste no se correlacionan con edema macular. Mansour, et al. describieron que la zona avascular foveal está aumentada significativamente en pacientes con MacTel tipo 2 y que la neovascularización subretiniana se desarrolla cerca de las zonas con migración de pigmento ${ }^{5}$.

En la OCT observamos asimetría en la depresión foveal y disrupción de la unión de los segmentos externos/internos de los fotorreceptores. Esta pérdida de fotorreceptores es previa a la aparición de los cambios capilares en la angiografía. Cuando progresa, se observan cavitaciones hiporreflécticas en la retina interna que se describen como agujeros pseudolamelares maculares. Estas progresan hacia la retina externa y provocan adelgazamiento de la mácula y atrofia ${ }^{6}$.

La angiografía por OCT (OCT-A) provee una evaluación tridimensional de la retina neurosensorial y la vasculatura retiniana, y nos permite un mapeo de los plexos vasculares no invasivo7. Por ello, un estudio combinado como la OCT-A sería ideal para estudiar las telangiectasias maculares tipo 2.

Se han descrito cinco estadios de la enfermedad según Gass $^{8}$, y se describen las características por OCT-A. En el estadio 1 se encuentran vasos telangiectásicos profundos localizados temporal a la zona avascular foveal (ZAF). En el estadio 2 se afectan los plexos superficial y profundo, hay una pérdida de densidad vascular e irregularidad en los capilares. En el estadio 3 la ZAF es irregular, hay invasión vascular al epitelio pigmentario de la retina (EPR). En el estadio 4 hay distorsión y arrastramiento vascular y ya se puede visualizar vascularización subretiniana. En el estadio 5 hay pérdida severa de la densidad vascular y membrana fibrovascular disciforme ${ }^{9}$.

En este estudio se reportan las características morfológicas de los capilares perifoveales, los plexos vasculares afectados y los cambios de la retina neurosensorial observados en la OCT-A en pacientes con MacTel tipo 2.

Adicionalmente se analizó si alguna de las variables encontradas en la OCT-A tiene correlación con la agudeza visual.

\section{Metodología}

Se trata de un estudio descriptivo, analítico y transversal donde se realizaron OCT-A en pacientes adultos (>18 años) diagnosticados con telangiectasias maculares tipo 2 mediante FAR, OCT o ambos. Se describieron los hallazgos encontrados en las imágenes y se analizaron para determinar si estos hallazgos tenían relación con la agudeza visual. Así mismo se determinó si los datos demográficos influían en la agudeza visual o en el estadio de la enfermedad. 
Se utilizó el equipo OCT Spectralis módulo OCT-A (Heidelberg Engeenering, Alemania) para la toma de OCT-A.

A todos los pacientes se les realizó el estudio en ambos ojos, escaneo de $3 \times 3 \mathrm{~mm}$ con un corte horizontal a nivel de la fóvea. Las imágenes fueron analizadas por ambos autores del presente estudio.

Únicamente se incluyeron pacientes con retinopatía diabética no proliferativa leve o moderada y sin afección macular secundaria a esta patología.

Se descartaron pacientes con patología vascular retiniana o macular por alguna otra causa.

\section{Resultados}

Se obtuvieron imágenes de OCT-A de 48 ojos de 24 pacientes con previo diagnóstico de MacTel tipo 2. Solo 43 ojos presentaron cambios consistentes con la patología tanto en la clínica como en FAR, OCT y OCT-A. El $70 \%$ fueron mujeres. El promedio de edad fue de 60 años (46 a 77 años). Se analizó si la edad se relacionaba con un estadio más avanzado de la enfermedad, pero no se encontró una correlación significativa $(p=0.13)$.

Como enfermedades sistémicas, el $45 \%$ padece hipertensión arterial sistémica (HAS) y el $29 \%$, diabetes mellitus tipo 2 (DM2); el resto niega patologías sistémicas. De los pacientes diabéticos, únicamente tres pacientes presentaban retinopatía diabética no proliferativa leve sin edema macular diabético, en el resto no se encontraron datos de retinopatía diabética.

Se analizaron estas comorbilidades y se observó que los pacientes diabéticos presentaban una agudeza visual significativamente peor $(p<0.05)$ que los pacientes con hipertensión arterial o sin patología sistémica.

Clínicamente, en el $100 \%$ de los ojos se observaron cambios hipopigmentados perifoveales; en el $35 \%$ de los ojos se observó una coloración grisácea perifoveolar, y el en $10 \%$ se observó un engrosamiento del área macular secundario a líquido subretiniano.

La agudeza visual promedio medida en LogMAR fue de 0.3 , lo que equivale a $20 / 40$ en la cartilla de Snellen (Tabla 1).

\section{Hallazgos en la angiografía por tomografía de coherencia óptica}

El $80 \%$ de los pacientes presentaron cambios en la OCT-A bilateral y el $20 \%$, en la unilateral.

Nueve ojos (21\%) presentaron neovascularización coroidea. Cuatro pacientes presentaron membrana neovascular franca, a los que se les indicó terapia con
Tabla 1. Agudeza visual mejor corregida (AVMC)

\begin{tabular}{|l|c|c|}
\hline AVMC & Número & $\%$ \\
\hline $20 / 20-20 / 40$ & 25 & 58 \\
\hline $20 / 50-20 / 80$ & 14 & 32 \\
\hline$<20 / 100$ & 4 & 10 \\
\hline
\end{tabular}

antiangiogénico intravítreo. Los cinco ojos restantes (55\%) presentaron neovascularización subclínica, ya que no se observaron datos de neovascularización por clínica, OCT ni FAR, solo se detectó en la capa avascular de la OCT-A (en estos pacientes se descartaron artefactos). Los pacientes con neovascularización subclínica no presentaban mayor afección visual (LogMAR 0.3) (Figs. 1 y 2).

También se tomó en cuenta la localización de los cambios vasculares en la OCT-A con respecto a la fóvea: el $63 \%$ (27) fueron en el cuadrante temporal, el $19 \%$ (8), en el superior, el $12 \%$ (5), en el nasal, y el $6 \%$ (3), en el inferior. Se analizó si la agudeza visual y el estadio se relacionaba con el cuadrante afectado, pero no se observó una correlación estadísticamente significativa ( $p=0.25$ y $p=0.31$, respectivamente).

El plexo vascular más afectado fue el profundo, en un $94 \%$. En el $35 \%$ de los pacientes se logró visualizar vasos en ángulo recto (Fig. 3). Las características morfológicas que observamos en los vasos afectados fueron: un engrosamiento con dilataciones focales y, solo en el $33 \%$, lesiones aneurismáticas con morfología (Fig. 3).

Según el estadiaje por OCT-A se encontró: el 21\% en estadio 1 , el $38 \%$ en estadio 2 , el $32 \%$ en estadio 3 y el $9 \%$ en estadio 4 . No se presentaron pacientes con características del estadio 5.

Los hallazgos más comunes en la retina neurosensorial por imagen de OCT macular fueron: puntos hiperreflécticos en el $86 \%$, discontinuidad complejo Bruch/ EPR y línea elipsoide en el $81 \%$, cavitaciones en el $54 \%$ y neovascularización coroidea en el $9 \%$ con engrosamiento macular por líquido intrarretiniano (Fig. 4). El grosor macular central (GMC) promedio fue de 236 um y no se encontró una relación significativa entre este y la agudeza visual $(p=0.29)$.

\section{Discusión}

En el Proyecto de MacTel, el estudio más grande de telangiectasias tipo 2, se encontró que los factores sistémicos más frecuentemente asociados son la diabetes 


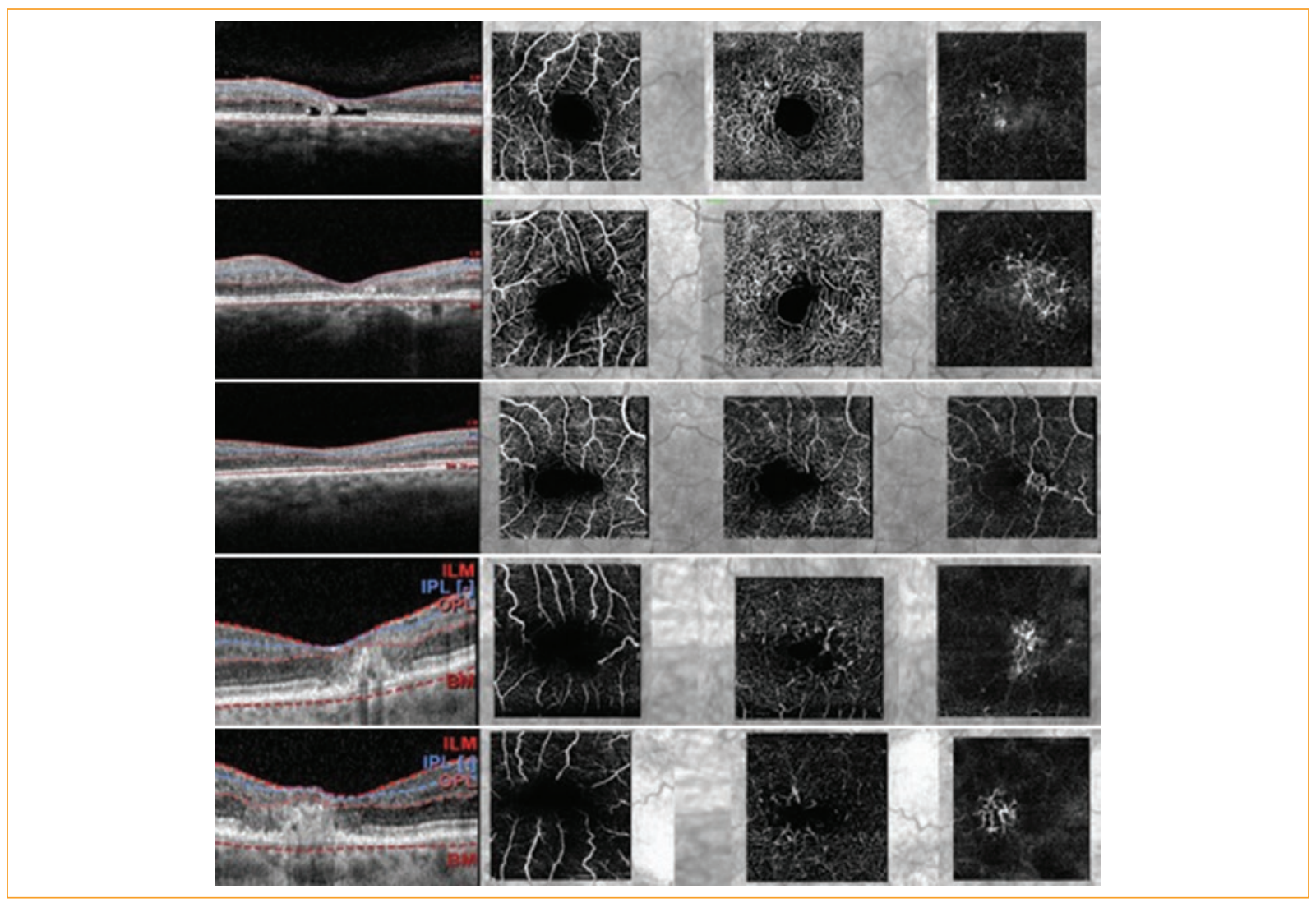

Figura 1. Imágenes de angiografía por tomografía de coherencia óptica de cinco pacientes con neovascularización evidenciada en el plexo avascular. La primera columna son imágenes de corte central macular, la segunda del plexo vascular superficial, la tercera del plexo profundo y la cuarta de la capa avascular.

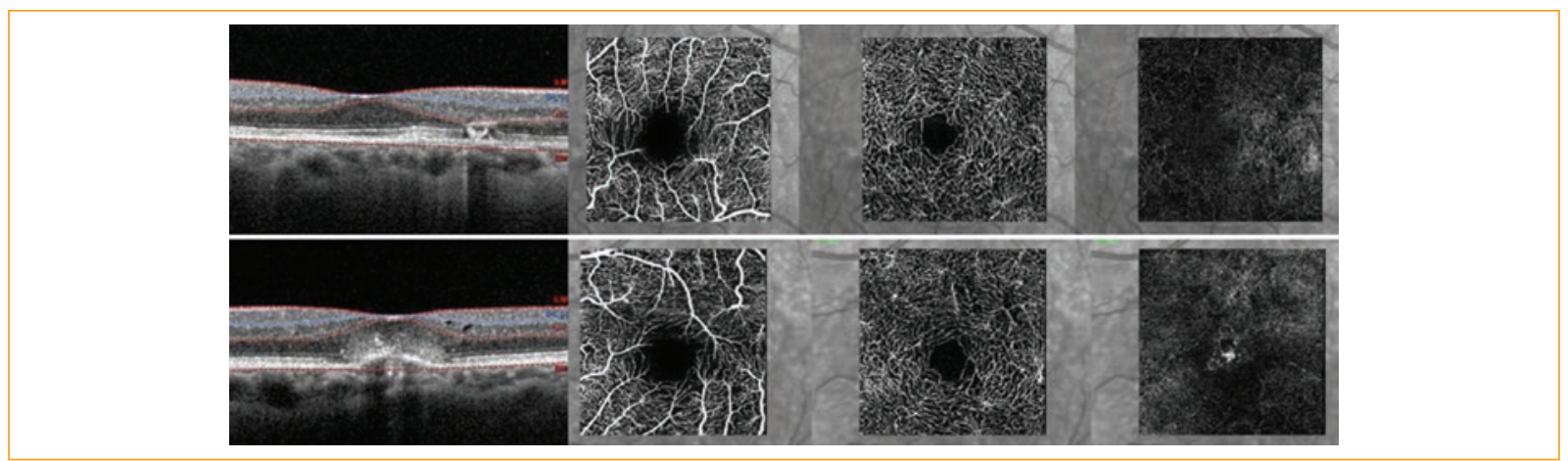

Figura 2. Imagen superior: angiografía por tomografía de coherencia óptica (OCT-A) del ojo derecho, plexo superficial con disminución de la densidad vascular leve, en el plexo profundo se observan vasos telangiectásicos perifoveales. Imagen inferior: OCT-A del ojo izquierdo, en el plexo superficial y profundo vasos telangiectásicos, pérdida de densidad vascular y en la capa avascular neovascularización.

(28\%) y la hipertensión arterial $(52 \%)^{10}$. En nuestro estudio pudimos observar que los pacientes con DM2 presentaban una agudeza visual significativamente peor que los pacientes con HAS o sin comorbilidades sistémicas. Podemos pensar que esto se atribuye al daño vascular que tienen de base los pacientes con DM2.
La edad no se determinó como un factor de riesgo para presentar una enfermedad más avanzada, ya que no existió correlación significativa.

Los hallazgos patológicos en su mayoría fueron bilaterales (80\%). Se observó una presentación asimétrica en general. Creemos que el $20 \%$ de los pacientes que 


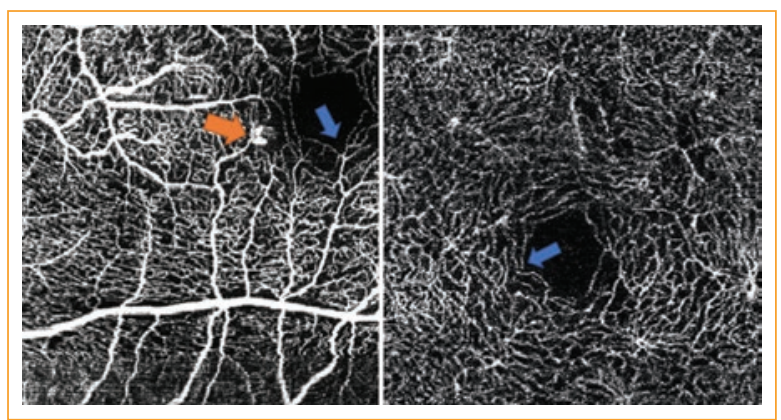

Figura 3. A la izquierda, angiografía por tomografía de coherencia óptica con lesión vascular temporal a la fóvea. Se observa lesión aneurismática en el plexo superficial (flecha naranja). Ambas imágenes con vasos en ángulo recto (flecha azul).

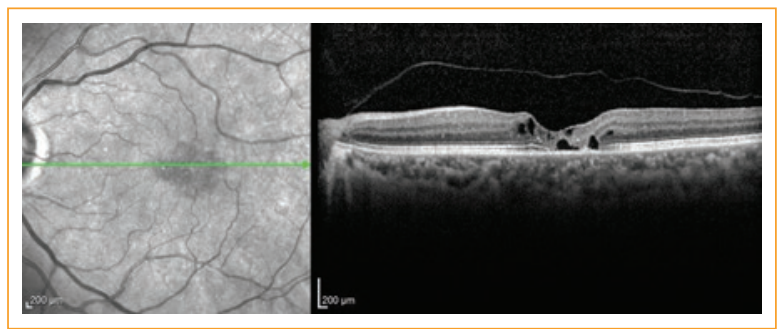

Figura 4. Características de retina neurosensorial. Se observan puntos hiperreflécticos, cavitaciones intrarretinianas, pérdida de la continuidad en la línea elipsoide y alteraciones del EPR.

solo presentaron cambios consistentes con MacTel tipo 2 (clínicamente, por OCT, FAR y OCT-A) en un solo ojo se debe a la asimetría de presentación de la enfermedad y no a una variedad unilateral del MacTel tipo 2.

La agudeza visual fue buena en general (20/40). Esto sorprende en MacTel tipo 2, ya que, en otras patologías donde la línea elipsoide se encuentra dañada, las agudezas visuales suelen ser peores.

En el Proyecto MacTel se reportó una prevalencia de neovascularización del $2 \%$ en las fotografías de fondo de ojo. Otro estudio más reciente encontró en las OCT-A invasión vascular en la retina externa y neovascularización en 9 de los 14 ojos estudiados ${ }^{11}$. En nuestro estudio, con el análisis de OCT-A, se encontró neovascularización en el $21 \%$. El $55 \%$ de estos pacientes presentaron neovascularización subclínica (solo en la OCT-A) y no se les ofreció tratamiento antiangiogénico, se mantienen en vigilancia estrecha.

Los cambios vasculares en su mayoría se presentaban en el área temporal y superior con respecto a la fóvea. No encontramos correlación en cuanto al cuadrante afectado y la agudeza visual.
Encontramos una presentación de la enfermedad en los diferentes estadios. El estadio más frecuente fue el 2 , seguido del estadio 3 (38 y $32 \%$, respectivamente).

Cabe recalcar que no se trata de una enfermedad meramente vascular. Ya se ha descrito que los cambios vasculares, en ocasiones, no son la característica prominente, sobre todo en estadios tempranos de la enfermedad. Gass describió cambios en la OCT, como agujeros lamelares, y en el electrorretinograma, una respuesta reducida de los conos antes de encontrar los cambios vasculares, lo cual refuerza la teoría de que la anormalidad primaria se encuentra en la retina neural y las células de Müller ${ }^{12}$. La anomalía más frecuente en la retina neurosensorial en nuestra serie de casos fue la pérdida de continuidad en la línea elipsoide y alteraciones de capas hiperreflécticas externas, puntos hiperreflécticos y cavitaciones. Los puntos hiperreflécticos nos sugieren una migración de células que intentan reparar el daño y un probable componente degenerativo más que vascular, ya que en otras patologías, como la retinosis pigmentaria, estos puntos se han descrito como lipoproteínas extravasadas, macrófagos y células del EPR que migran. Quizá los cambios vasculares sean secundarios al daño de la retina neurosensorial ${ }^{13}$. Apoyamos esta teoría con los estudios de nuestros pacientes, donde los cambios de la retina neurosensorial eran más evidentes que los cambios vasculares de OCT-A.

Heeren, et al. observaron que la agudeza visual no es necesariamente la mejor medida del deterioro funcional. Ellos midieron la agudeza visual y la microperometría. De 71 pacientes, el $58 \%$ de los ojos la microperimetría demostraba aumento en el escotoma o un nuevo escotoma absoluto. En cambio, solo el 17\% de estos pacientes tenía disminución de la agudeza visual de más de dos líneas ${ }^{14}$.

Finger, et al. reportaron que la pérdida sensitiva parafoveal se asociaba a dificultad para la lectura a pesar de una fijación central estable ${ }^{15}$.

Runkle, et al. realizaron OCT-A en 14 ojos y observaron una disminución del $50 \%$ en la densidad vascular con respecto a los controles, pero concluyeron que la disminución de la densidad vascular no se correlacionaba con la agudeza visual. También midieron el grosor de la zona elipsoide y el EPR, y vieron que era significativamente menor con relación a los controles y que este daño si se relacionaba a una menor agudeza visual ${ }^{16}$.

MacTel tipo 2 es una enfermedad subdiagnosticada, sobre todo en etapas tempranas, debido a que los cambios no son muy evidentes en la exploración y no causan una baja visual importante. En etapas tardías 
también es difícil su diagnóstico por su semejanza con la degeneración macular relacionada a la edad.

Charbel, et al. describieron que el grosor macular central se encontraba disminuido a expensas de contracción y atrofia de las capas externas de la retina; y que este factor se asociaba con datos de progresión de la enfermedad ${ }^{17}$. Nosotros no encontramos correlación significativa entre el GMC y la agudeza visual.

En nuestro estudio evidenciamos la utilidad de la OCT y su modalidad de OCT-A, ya que esta nos puede demostrar cambios discretos e iniciales en la evolución de la enfermedad, en especial para evidenciar neovascularización temprana, a los cuales se les debe realizar una vigilancia más estrecha. No creemos necesarios estudios con contraste como la fluorangiografía en el diagnóstico de MacTel tipo 2, ya que no nos ofrece información adicional a la que nos brinda el OCT-A.

\section{Conclusiones}

La MacTel tipo 2 es una patología compleja. La OCT-A nos muestran los cambios característicos en la retina neurosensorial y en los diferentes plexos capilares, sin la necesidad de otros estudios. La mayoría de los pacientes tienen una agudeza visual mejor a 20/40. La mayoría se encuentran en un estadio 2 y 3 según los hallazgos por OCT-A. No encontramos relación entre nuestras variables y la agudeza visual de los pacientes con este diagnóstico. Los pacientes diabéticos con MacTel tipo 2 presentan peores agudezas visuales. Los datos patológicos son más evidentes en la retina neurosensorial que los cambios vasculares, lo que apoya un origen celular con afección vascular secundaria. Así mismo, la OCT-A es el estudio ideal para detectar neovascularización temprana en esta patología. Los biomarcadores en OCT-A son difíciles de determinar para el pronóstico visual.

\section{Conflicto de intereses}

Todos los autores declaran la inexistencia de conflicto de intereses.

\section{Financiación}

Los autores declaran que no recibieron financiación para esta publicación.

\section{Responsabilidades éticas}

Protección de personas y animales. Los autores declaran que para esta investigación no se han realizado experimentos en seres humanos ni en animales.

Confidencialidad de los datos. Los autores declaran que han seguido los protocolos de su centro de trabajo sobre la publicación de datos de pacientes.

Derecho a la privacidad y consentimiento informado. Los autores han obtenido el consentimiento informado de los pacientes y/o sujetos referidos en el artículo. Este documento obra en poder del autor de correspondencia.

\section{Bibliografía}

1. Klein R, Blodi B, Meuer S Myers CE, Chew EY, Klein BE. The prevalence of macular telangiectasia type 2 in the Beaver Dam Eye study. Am J Ophtalmol. 2010;150:55-62.

2. Sallo F, Leung I, Mathenge W, Kyari F, Kuper H, Gilbert CE, et al. The prevalence of type 2 idiopathic macular telangiectasias in two African populations. Opthalmic Epidemiol. 2012;19:185-9.

3. Green W, Quigley H, De la Cruz Z, Cohen B. Parafoveal retinal telangiectasias. Light and electron microscopy studies. Trans Ophthalmol Soc UK. 1980:100:162-70.

4. Gass JD. Müller cell cone, an overlooked part of the anatomy of the fovea centralis: Hypotheses concerning its role in the pathogenesis of macular hole and retinoschisis. Arch Opthalmol 1999;117:660-73.

5. Mansour A, Schachat A. Foveal avascular zone in idiopathic parafoveal telangiectasia. Ophthalmologica. 1993;207:9-12.

6. Gupta V, Gupta A, Dogra M, Agarwal A. Optic coherence tomography findings in group $2^{\underline{a}}$ idiopathic yuxtafoveolar telangiectasias. Ophthalmic Surg Laser Imaging. 2005;36:482-6

7. De Carlo T, Romano A, Waheed N, Duker J. A review of optical coherence tomography angiography (OCTA). Int J Retin Vitr. 2015;1(1):5

8. Gass J, Blodi B. Idiopathic juxtafoveal telangiectasis. Update of classification and follow-up study. Ophthalmology. 1993;100:1536-46.

9. Thorell W, Zhang Q, Huang $Y$, An L, Durbin MK, Laron M, et al. Swept-Source OCT Angiography of Macular Telangiectasia Type 2, Ophthalmic Surgery, Lasers, and Imaging Retina. 2014;45(5):369-80.

10. Clemons T, Gillies M, Chew E, Bird AC, Peto T, Figueroa MJ, et al. Baseline characteristics of participants in the natural history study of macular telangiectasia MacTel Project Report No.2. Ophthalmic Epidemiol. 2010;17:66-73.

11. Spaide R, Klancnik J, Cooney M. Retinal Vascular Layer in Macular Telangiectasias Type 2 Imaged by Optical Coherence Tomographic Angiography. JAMA Ophthalmol. 2015;133(1):66-73.

12. Gass JDM. Histological study presumed parafoveal telangiectasia. Retina 2000:20:266-7.

13. Kuroda M, Hirami $Y$, Hata M, Mandai M, Takahashi M, Kurimoto Y. Intraretinal hyperreflective foci on spectral-domain optical coherence tomographic images of patients with retinitis pigmentosa. Clin Ophthalmol. 2014:8:435-40.

14. Heeren TF, Clemons T, Scholl HP, Bird AC, Holz FG, Charbel Issa P. Progression of visión loss in macular telangiectasias type 2. Invest Ophthalmol Vis Sci. 2015;56:3905-12.

15. Finger R, Charbel Issa P, Fimmers R, Holz FG, Rubin GS, Scholl HP. Reading performance is reduced by parafoveal escotomas in macular telangiectasia type 2. Invest Ophthalmol Vis Sci. 2009;50:1366-70.

16. Runkle AP, Kaiser PK, Srivastava SK, Schachat AP, Reese JL, Ehlers JP. OCT Angiography and Ellipsoid Zone Mapping of Macular Telangiectasia Type 2 From AVATAR Study. Invest Ophthalmol Vis Sci. 2017;58:3683-9.

17. Charbel Issa $P$, Gillies MC, Chew EY, Bird AC, Heeren TF, Peto T, et al. Macular telangiectasias type 2. Prog Retin Eyes Res 2013;34:49-77. 